\title{
Vibrational Energy Redistribution in a Gas-Surface Encounter: State-to-State Scattering of $\mathrm{CH}_{4}$ from Ni(111)
}

\author{
Jörn Werdecker, Maarten E. van Reijzen, Bo-Jung Chen, and Rainer D. Beck* \\ Laboratoire de Chimie Physique Moléculaire, École Polytechnique Fédérale de Lausanne, CH-1015 Lausanne, Switzerland
}

(Received 29 September 2017; published 31 January 2018)

\begin{abstract}
The fate of vibrational energy in the collision of methane $\left(\mathrm{CH}_{4}\right)$ in its antisymmetric $\mathrm{C}-\mathrm{H}$ stretch vibration $\left(\nu_{3}\right)$ with a $\mathrm{Ni}(111)$ surface has been studied in a state-to-state scattering experiment. Laser excitation in the incident molecular beam prepared the $J=1$ rotational state of $\nu_{3}$, and a bolometer in combination with selective laser excitation detected the scattered methane. The rovibrationally resolved scattering distributions reveal very efficient vibrational energy redistribution from $\nu_{3}$ to the symmetric $\mathrm{C}-\mathrm{H}$ stretch vibration $\left(\nu_{1}\right)$. The branching ratio $\nu_{1} / \nu_{3}$ is near 0.4 and insensitive to changes in incident kinetic energy in the range from 100 to $370 \mathrm{meV}$. State-resolved angular distributions and measurements of the residual Doppler linewidths prove that the scattering is direct. The observed vibrationally inelastic scattering provides direct experimental evidence for surface-induced vibrational energy redistribution.
\end{abstract}

DOI: 10.1103/PhysRevLett.120.053402

The variety of dynamical processes that occur when molecules interact with solid surfaces are of fundamental interest, as they all play their part in the complex chemical reaction mechanisms found in heterogeneous catalysis. Over the last decades, quantum state-resolved molecular beam scattering from well-defined surfaces has contributed much to our understanding of these dynamics [1-6]. Apart from giving direct insight, state-resolved data are used to gauge theoretical models, thereby paving the way toward a predictive understanding of heterogeneous catalysis [7-11]. Experiments that probe the quantum state populations of the scattered products have proven particularly rewarding as the final distributions resolved in internal quantum state, angle, and speed contain a wealth of dynamical information.

Because of the wide dispersion of scattering products in space, time, and internal state, experimentalists have so far relied mostly on the very sensitive resonantly enhanced multiphoton ionization (REMPI) method for state-resolved detection. However, the systems accessible with REMPI or LIF are with few exceptions [12-15] limited to atoms and diatomic molecules. Consequently, previous surface scattering studies have mainly focused on these species.

By contrast, studies that probe the postscattering state distributions of polyatomic molecules remain scarce [12-20]. This is unfortunate since the dynamics of polyatomics at surfaces are expected to be rich and distinctly different from those of diatomics, not least due to the additional vibrational degrees of freedom (d.o.f.) [21]. For example, one might ask about the interactions between the different vibrational modes and the normal-to-local-mode evolution as the molecule approaches the surface. Closely related are questions concerning the flow of vibrational energy between modes [22]: can the surface interaction enable such intramolecular vibrational energy redistribution (IVR) and is IVR fast enough to control the reactivity on the surface? Information on the fate of initial energy can be obtained in a state-to-state scattering experiment, which both prepares the incoming molecule in one specific state and analyzes the states of the scattered species.

The combination of a bolometer as a molecular beam detector with modulated infrared (IR) laser excitation provides [23] an alternative to the aforementioned stateselective probes. The bolometer is a thermal detector responding to the kinetic and internal energy that the molecular beam gives up when it impinges on the detector. A modulated IR laser excites ("tags") molecules on their way to the bolometer, giving rise to an ac signal due to the added rovibrational energy. Selective amplification of this ac component removes all contributions from the bolometer signal, which are unrelated to the laser excitation, and gives a direct measure of the transferred population.

The technique was used by Miller and co-workers who performed a series of scattering experiments with methane $\left(\mathrm{CH}_{4}\right)$ and acetylene [17-20]. They illustrate the advantage that any state can be probed that is accessible by IR-active transitions. However, the absence of adequate high-power IR lasers in the past has prevented a widespread use as a scattering probe. Since then, a new class of high-power, continuous-wave (cw), single-mode IR light sources based on fiber laser pumped optical parametric oscillators (OPOs) has become available [24]. Their high brightness and coherence has enabled sophisticated laser pumping schemes. Specifically, rapid adiabatic passage (RAP) can routinely achieve near complete population inversion in a molecular beam $[25,26]$.

In the present work, we applied bolometric detection in combination with highly efficient IR laser pumping via 
RAP to investigate the fate of initial vibrational energy in the surface scattering of methane, a molecule for which no REMPI scheme is known. This Letter reports the first stateto-state molecular beam-surface scattering of $\mathrm{CH}_{4}$ from $\mathrm{Ni}(111)$. Our work is motivated by results from previous studies $[4,6,9,27]$ on the dissociative adsorption of $\mathrm{CH}_{4}$ on nickel, which has received much attention due to its importance as rate-limiting step in the steam-reforming reaction.

Previous state-resolved reactivity measurements have shown that the partitioning of internal energy can influence the probability and products of the dissociation reaction. Specifically, one quantum of symmetric $\mathrm{C}-\mathrm{H}$ stretch excitation $\left(\nu_{1}\right)$ promotes the $\mathrm{C}$-H bond cleavage on $\mathrm{Ni}(100)$ up to ten times more effectively than a quantum of antisymmetric $\mathrm{C}-\mathrm{H}$ stretch excitation $\left(\nu_{3}\right)$, even though the two vibrations have comparable energies of 2916 and $3019 \mathrm{~cm}^{-1}$, respectively $[28,29]$. This nonstatistical outcome suggests that surface-induced IVR [22] is incomplete. Deeper insight into the role of IVR from experiment is desirable, as its treatment is an aspect that distinguishes current theoretical treatments of this prototypical gas-surface reaction.

The molecular beam-surface science apparatus without the bolometer has been described previously [30]. A molecular beam was generated by expanding pure $\mathrm{CH}_{4}$ or mixtures with helium. The speed distributions of the beams were determined using the time-of-flight method. The scattering target was a $10 \mathrm{~mm}$ diameter $\mathrm{Ni}(111)$ surface. All experiments were carried out with a surface temperature of $T_{S}=673 \mathrm{~K}$, which keeps the carbon solubility in nickel high enough to avoid buildup of surface carbon due to background gas or chemisorbed $\mathrm{CH}_{4}$. Details of the molecular beam conditions and the surface sample can be found in the Supplemental Material [31].

Incident $\mathrm{CH}_{4}$ was state prepared with one quantum of antisymmetric stretch vibration $\left(\nu_{3}\right)$ and in rotational state $J=1$ via the $\nu_{3} \leftarrow v=0, R(0)$ transition using the idler output $(\sim 2 \mathrm{~W})$ from a tunable cw IR OPO (Pump OPO). The laser beam is focused by a cylindrical lens $(f=25 \mathrm{~cm})$ before intersecting the incident molecular beam in order to enable RAP excitation, which affords almost complete (typically $~ 94 \%$ for the pumping step) inversion of population [26,33].

A detailed description of the bolometer and laser tagging setup will be published elsewhere. Briefly, we added a cryogenic bolometer to the main surface science chamber of our apparatus making an angle of $135^{\circ}$ with the incident molecular beam, as depicted in Fig. 1. The detector is a doped silicon bolometer, mounted to a liquid helium bath cryostat held at $1.6 \mathrm{~K}$ using evaporative cooling. A thin $4 \mathrm{~mm}$ diameter diamond disk bonded to the bolometer element provides a surface for the adsorption of scattered molecules. They are tagged before reaching the bolometer using the idler output $(\sim 0.7 \mathrm{~W})$ from a second IR OPO ("Tagging OPO"). The laser beam is directed to an optical access port upstream of the bolometer and focused by a $f=25 \mathrm{~cm}$ cylindrical lens to intersect the scattered

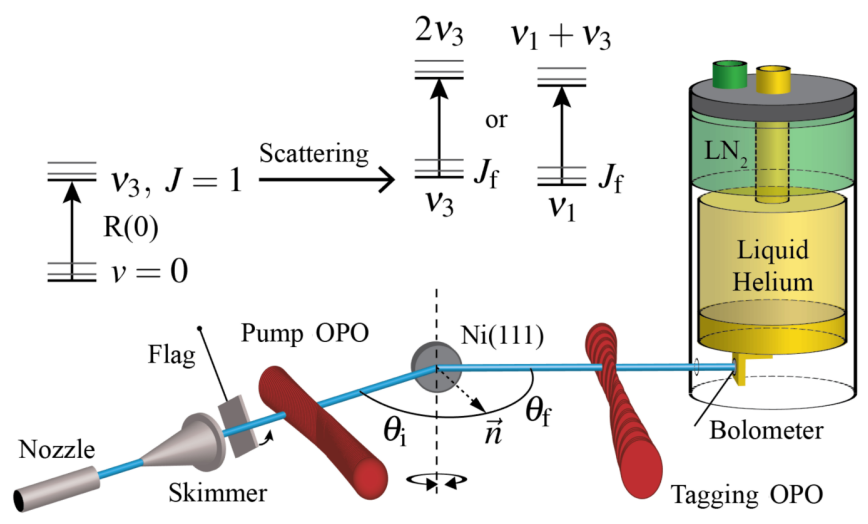

FIG. 1. Schematic diagram of the experimental apparatus (not to scale). A continuous-wave IR OPO ("Pump OPO") prepares a continuous molecular beam of $\mathrm{CH}_{4}$ in the $\left(\nu_{3}, J=1\right)$ state before the beam hits a $\mathrm{Ni}(111)$ surface at an angle $\theta_{i}$ measured from the surface normal $\vec{n}$. Scattered products are detected under an exit angle $\theta_{f}=135^{\circ}-\theta_{i}$ using a bolometer after selective excitation by a second but modulated (chopped) IR OPO. A flag can be moved to block the incoming molecular beam. (Top left) Illustration of the laser excitation scheme. $\mathrm{CH}_{4}\left(\nu_{3}, J=1\right)$ is scattered into final rotational states $J_{f}$ and detected in either $\nu_{3}$ or $\nu_{1}$ by laser excitation to the first overtone of $\nu_{3}$ or the $\nu_{1}+\nu_{3}$ combination band, respectively.

molecules and likewise achieve RAP conditions. Both lasers have instrumental linewidths of $1.0 \pm$ $0.5 \mathrm{MHz}$ (FWHM).

If the tagging laser frequency $\omega_{2 \leftarrow 1}$ is resonant with a molecular transition $|2\rangle \leftarrow|1\rangle$, a fraction $f_{\text {tag }}$ of the scattered molecules is excited. The laser beam is modulated with a chopper wheel and the corresponding ac part of the bolometer signal is selectively amplified using a lock-in amplifier. The demodulated signal $S_{\text {tag }}$ is related to the flux $F_{1}$ of molecules that reside in state $|1\rangle$ by $S_{\text {tag }} \propto F_{1} \times$ $f_{\text {tag }} \times \hbar \omega_{2 \leftarrow 1}$. Because neither thermal nor collisional energies are sufficient to populate the upper tagging levels, located $\sim 6000 \mathrm{~cm}^{-1}$ above the ground state, the tagging laser only excites molecules from $|1\rangle$ to $|2\rangle$. Excitation by RAP avoids population return to $|1\rangle$ caused by stimulated emission and can achieve almost full population inversion. We determine the background signal coming from scattered laser light by temporarily blocking the molecular beam using a beam flag and subtract it from the flag-open signal.

Methane is a spherical top and occurs in three nuclear spin modifications due to its four equivalent hydrogen atoms [34]. We prepare and detect $m e t a-\mathrm{CH}_{4}$ (nuclear spin quintet), owing to its favorable statistical weight. Rotational state populations of the $\nu_{3}$ and $\nu_{1}$ vibrational states after scattering were probed by tagging via $2 \nu_{3} \leftarrow \nu_{3}$ and $\nu_{1}+$ $\nu_{3} \leftarrow \nu_{1}$ transitions, respectively. In both cases, we used either $R$ - or $Q$-branch transitions depending on transition strength and wavelength accessibility. Except for the strongest $2 \nu_{3} \leftarrow \nu_{3}$ transitions, for which population inversion was readily achieved, we always measured the dependence of $S_{\operatorname{tag}}$ on the laser fluence. We fitted the 
fluence curves using an empirical model based on the Landau-Zener formulation for RAP excitation [26], as detailed in the Supplemental Material [31]. The curve asymptotes, which correspond to $f_{\text {tag }}=1$, were used as a measure of state populations, thereby making a correction for transition strengths superfluous. We only corrected for the respective photon energy $\hbar \omega_{2 \leftarrow 1}$. Rotational state distributions were recorded at an incidence angle of $\theta_{i}=$ $65^{\circ}$ and a final angle of $\theta_{f}=70^{\circ}$. This geometry avoids the maximum of the specularly reflected helium, which is intense enough to cause signal instabilities and reduction of bolometer sensitivity.

Figure 2 shows the rotational state distributions after scattering where the incident methane was in the $\left(\nu_{3}, J=1\right)$ state and had a kinetic energy of $E_{i}=230 \mathrm{meV}$. We observe two main scattering channels, the first being the vibrationally elastic channel, where $\mathrm{CH}_{4}$ remains in its initial vibrational state $\left(\nu_{3} \rightarrow \nu_{3}\right)$. Second, we identify a vibrationally inelastic channel, where the vibrational energy has been redistributed into the symmetric stretch $\left(\nu_{3} \rightarrow \nu_{1}\right)$. For both channels, we observe significant rotational excitation. Note that rotational levels do not exist for certain values of $J$ due to symmetry restrictions [34]. Our rotational distributions include all existing levels from $J=0$ through $J=8$, except for one sublevel belonging to $\left(\nu_{3}, J=8\right)$, which was most likely below the detection limit. We verified that there is no thermally or collisionally induced excitation from $v=0$ to $\nu_{1}$ and $\nu_{3}$. All observed products therefore originate solely from scattering of $\mathrm{CH}_{4}\left(\nu_{3}, J=1\right)$.

The rotational distributions for incidence energies of 100 and $370 \mathrm{meV}$ are similar to those in Fig. 2. The total flux in each vibrational scattering channel is the combined intensity of all rotational sublevels belonging to the corresponding vibrational state. The branching ratio between the two vibrations $b_{\nu_{1} / \nu_{3}}$ is then given by

$$
b_{\nu_{1} / \nu_{3}}=\frac{S_{\nu_{1}}^{\text {total }}}{S_{\nu_{3}}^{\text {total }}}=\frac{\sum_{J=0}^{8} \sum_{n \in J} S_{\nu_{1}, J, n}}{\sum_{J=1}^{8} \sum_{n \in J} S_{\nu_{3}, J, n}},
$$

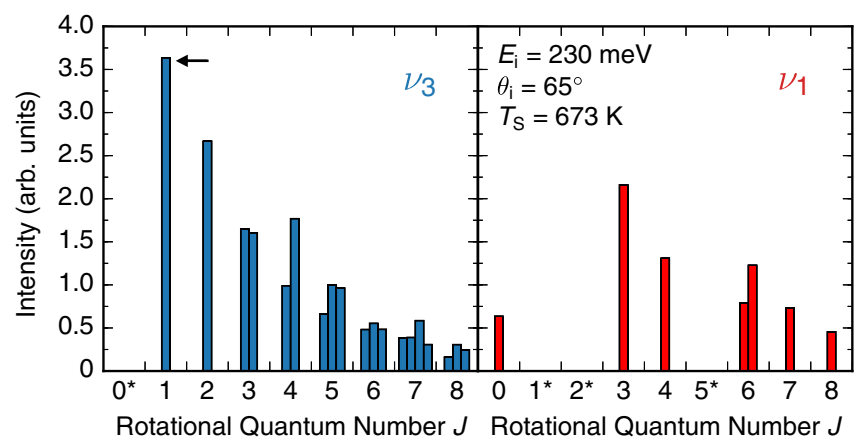

FIG. 2. Rotational state distributions within the vibrationally elastic $\left(\nu_{3}\right)$ and inelastic $\left(\nu_{1}\right)$ channels for a molecular beam of $\mathrm{CH}_{4}\left(\nu_{3}, J=1\right)$ scattered from $\mathrm{Ni}(111)$ with an average incidence translational energy of $230 \mathrm{meV}$. All bars represent individual rotational sublevels. Asterisks mark nonexisting levels and the arrow highlights the initial state. where the second summation is, respectively, carried out over all sublevels $n$ belonging to the same value of $J$. We find a branching ratio near $40 \%$, which is insensitive to $E_{i}$ across the studied energy range (see Table I). Other scattering channels, which may populate bend overtones $\left(2 \nu_{2}, 2 \nu_{4}\right)$ or the $\nu_{2}+\nu_{4}$ level, are the subject of future study. However, we can already say that the probability of populating one of the these states is lower by at least a factor of 5 compared to that for $\nu_{1}$. Furthermore, we observe no population in the fundamental bend vibrations $\nu_{2}\left(1533 \mathrm{~cm}^{-1}\right)$ and $\nu_{4}\left(1311 \mathrm{~cm}^{-1}\right)$.

Figure 3(a) displays a Boltzmann plot of the distributions from Fig. 2. The distribution corresponding to $\nu_{3}$ is nonBoltzmann, showing a propensity for rotationally elastic scattering. For $J \geq 3$, the distribution falls approximately on a straight line in this plot. Boltzmann analyses of the linear regimes yield rotational temperatures $T_{\text {rot }}$ between 140 and $200 \mathrm{~K}$, far below the surface temperature of $673 \mathrm{~K}$, which is consistent with direct scattering. It stands in contrast to a possible trapping-desorption (TD) mechanism, in which molecules become trapped for some time in a weakly bound (physisorbed) state and subsequently desorb with their d.o.f. accommodated to the surface temperature. Hence, if the scattering were dominated by TD, rotational temperatures closer to $T_{S}$ would be expected. Table I compiles the rotational temperatures for all $E_{i}$. Rotational excitation increases slightly with $E_{i}$, which is likewise expected for direct scattering. Interestingly, rotational excitation is slightly higher for $\nu_{1}$ than $\nu_{3}$ in all cases. We quantify this difference in terms of the mean rotational energy difference $\Delta\left(E_{\text {rot }}\right)_{\nu}=\left\langle E_{\text {rot }}^{\nu_{1}}\right\rangle-\left\langle E_{\text {rot }}^{\nu_{3}}\right\rangle$ with

$$
\left\langle E_{\text {rot }}^{\nu}\right\rangle=\frac{1}{S_{\nu}^{\text {total }}} \sum_{J=0}^{8} \sum_{n \in J} S_{\nu, J, n}\left(E_{\nu, J, n}-E_{\nu, J=0}\right),
$$

where $E_{J, n}$ is the energy of a rotational sublevel and $E_{J=0}$ is that of the $J=0$ state. For $\nu_{3}$, we used the expected energy of a hypothetical $J=0$ level. The difference in $\left\langle E_{\text {rot }}\right\rangle$ accounts for nearly $40 \%$ of the energy $\Delta E_{\mathrm{vib}}=103 \mathrm{~cm}^{-1}$ liberated upon $\nu_{3} \rightarrow \nu_{1}$ conversion. The remainder of $\Delta E_{\text {vib }}$ is most likely converted into translational energy, as previous studies have shown that the reverse process, translational to vibrational energy transfer, can be efficient $[12,14]$.

Figure 3(b) shows state-resolved angular distributions for both scattering channels obtained by rotating the surface about an axis perpendicular to the scattering plane. The

TABLE I. Dependence of the branching ratios $b_{\nu_{1} / \nu_{3}}$, rotational temperatures, and difference in mean rotational energy on $E_{i}$. The two lowest $J$ levels were omitted in the determination of $T_{\text {rot }}^{\nu_{3}}$ (see text).

\begin{tabular}{lcccc}
\hline \hline$E_{i}(\mathrm{meV})$ & $b_{\nu_{1} / \nu_{3}}$ & $T_{\text {rot }}^{\nu_{3}}(\mathrm{~K})$ & $T_{\text {rot }}^{\nu_{1}}(\mathrm{~K})$ & $\Delta\left(E_{\mathrm{rot}}\right)_{\nu}\left(\mathrm{cm}^{-1}\right)$ \\
\hline 100 & $0.41 \pm 0.04$ & $153 \pm 8$ & $161 \pm 14$ & $37 \pm 15$ \\
230 & $0.39 \pm 0.06$ & $162 \pm 11$ & $178 \pm 17$ & $40 \pm 15$ \\
370 & $0.43 \pm 0.05$ & $181 \pm 13$ & $190 \pm 20$ & $41 \pm 15$ \\
\hline \hline
\end{tabular}



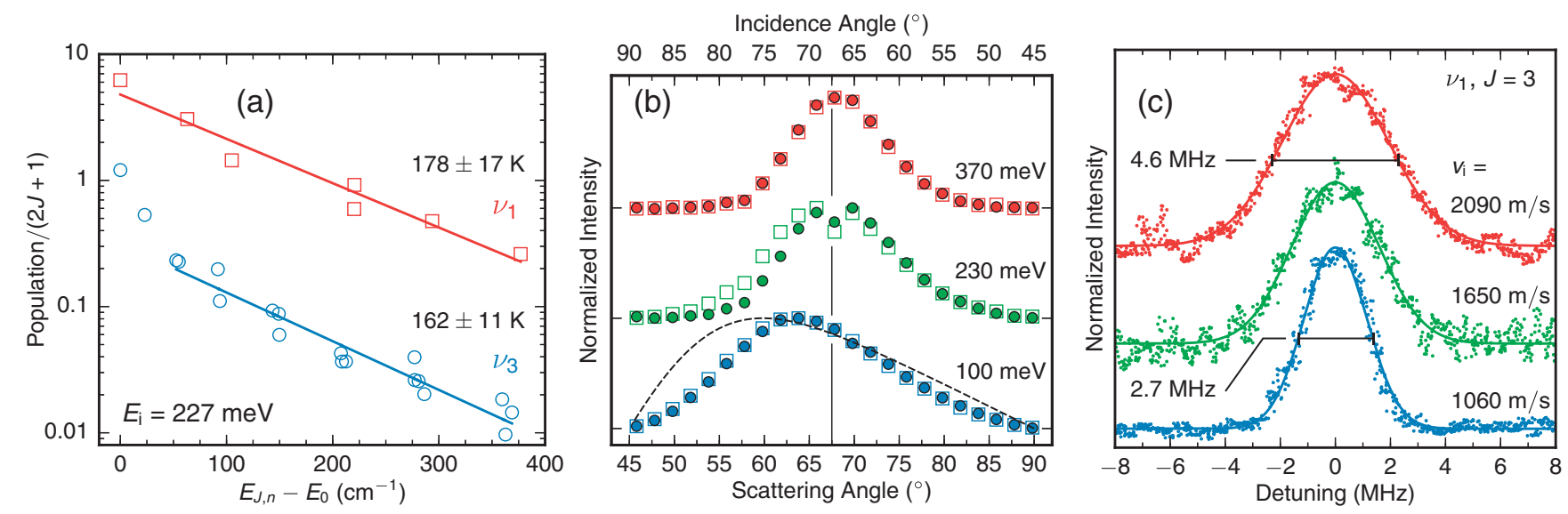

FIG. 3. (a) Rotational Boltzmann plot for the distributions in Fig. 2 with $\nu_{1}$ data (squares) scaled by a factor of 10 for visibility. $E_{0}$ is the energy of the lowest rotational state accessible for $\mathrm{CH}_{4}$ in the $\nu_{3}$ and $\nu_{1}$ vibrational states; $J=1$ and $J=0$, respectively. Solid lines are linear fits to the data, where for $\nu_{3}$ the two lowest levels were excluded from the analysis. Indicated are the characteristic rotational temperatures $T_{\text {rot }}$ obtained from the slopes. (b) Scattering angular distributions, normalized with respect to the peak value. Open and filled symbols represent $\mathrm{CH}_{4}$ in the $\left(\nu_{1}, J=3\right)$ and $\left(\nu_{3}, J=2\right)$ states, respectively. The three pairs of distributions are vertically offset from each other for clarity. Horizontal ticks indicate the zero levels. The vertical line depicts the specular angle and the dashed line shows the prediction for a pure $\cos \left(\theta_{f}\right)$ distribution. (c) Scans of the Doppler broadened absorption profile of the $\nu_{1}+\nu_{3} \leftarrow \nu_{1} R(3)$ transition for three incidence speeds $v_{i}$, corresponding to $E_{i}=100,230$, and $370 \mathrm{meV}$. Solid lines are Gaussian fits guiding the eye.

specular angle $\left(\theta_{i}=\theta_{f}\right)$ is indicated by a vertical line. All distributions are narrower than a $\cos \left(\theta_{f}\right)$ distribution, which would be expected for a pure TD mechanism. The predicted $\cos \left(\theta_{f}\right)$ curve shown in Fig. 3(b) includes the convolution with the experimental geometry, which leads to a dropoff toward smaller scattering angles, where the surface assumes a parallel orientation with respect to the incident beam. The dip observed close to the specular angle for $E_{i}=230 \mathrm{meV}$ comes from sensitivity reduction due to overlap with the reflected He beam. A lower He concentration was used for $E_{i}=370 \mathrm{meV}$, explaining the absence of a similar dip. Only at $E_{i}=100 \mathrm{meV}$, we see the distribution shifted toward the subspecular direction $\left(\theta_{f}<67.5^{\circ}\right)$. This is expected because of the dominance of phonon annihilation over creation when a slow projectile is scattered from a hot surface $[35,36]$. With increasing $E_{i}$, the distributions become narrower and peak closer to the specular angle, consistent with direct scattering. The strong similarity of the $\nu_{3}$ and $\nu_{1}$ angular distributions together with the insensitivity of the branching ratio to $E_{i}$ support the conclusion that, even if TD or multibounce trajectories were important, they exhibit no increased probability for vibrationally inelastic scattering.

We find further evidence for direct scattering in the tagging transition line shapes. They are shown in Fig. 3(c) for the $\nu_{1}+\nu_{3} \leftarrow \nu_{1} R(3)$ transition and the three incidence beam energies. These scans were carried out without the cylindrical lens in place and with reduced laser power to avoid saturation. In our current experimental geometry, residual Doppler broadening from the small velocity component along the laser propagation direction limits the transition linewidths. Although the resolution is not sufficient to extract the final speed distributions from the line shapes, the correlation between incidence speed and Doppler linewidths shows that the scattered molecules retain a memory of the incidence speed. Such a memory would be lost rapidly if the molecules experienced multiple bounces on the surface. Recent results by Al Taleb and Farías show that, even at $E_{i}$ as low as $69 \mathrm{meV}$, direct scattering dominates over TD [37]. Unlike in their angular distributions, we do not observe sharp diffraction peaks. The difference likely comes from the higher surface temperature we use, leading to stronger Debye-Waller attenuation.

Our results show that vibrational energy redistribution from the antisymmetric to the symmetric $\mathrm{C}-\mathrm{H}$ stretch vibration is very efficient. It is well established that IVR in isolated gas molecules and in solution is initiated by rapid energy transfer among near resonant "doorway" states from which slower IVR channels lead to a full redistribution of vibrational energy across the whole density of states [38]. Moore and co-workers observed similar behavior in the collisional deactivation of vibrationally excited $\mathrm{CH}_{4}$ in the gas phase [39,40]. These authors deduced a rapid equilibration of population among the $\nu_{3}$ and $\nu_{1}$ modes before these states decay further. Notably, they also found a four times lower stretch to bend overtone conversion rate compared to the $\left(\nu_{3} \leftrightarrow \nu_{1}\right)$ rate, consistent with our observations. This might seem surprising considering that the $2 \nu_{2}$ bend state is only $44 \mathrm{~cm}^{-1}$ higher in energy than $\nu_{3}$ and therefore energetically closer than $\nu_{1}$. However, one might think of the difference between the two stretching modes as being due to a phase shift of two of the C-H oscillators. It is conceivable that a collision can give rise to this phase shift more readily than to a complex deformation motion. Another possible explanation is that the excess vibrational energy can be 
accommodated by the rotational d.o.f. in the case of $\nu_{3} \rightarrow$ $\nu_{1}$ conversion, whereas $\nu_{3} \rightarrow 2 \nu_{2}$ requires energy transfer from the surface.

The current results clearly show that selective vibrational excitation of incident $\mathrm{CH}_{4}$ gives rise to highly nonstatistical product distributions in direct scattering. This finding is complementary to the previously observed mode specificity in the dissociative adsorption of $\mathrm{CH}_{4}$ on $\mathrm{Ni}$ surfaces. Some theoretical work aimed at describing this nonstatistical reactivity and calculated the vibrational mode evolution as the molecule approaches the transition state to reaction $[27,41]$. The calculations suggest a coupling of the $\nu_{1}$ and $\nu_{3}$ modes due to the surface interaction, which could give rise to vibrational energy flow during the approach [27,42]. The results presented in this Letter provide direct evidence for this surface-induced IVR.

In conclusion, we have performed the first state-to-state scattering experiments for vibrationally excited methane colliding with a Ni(111) surface. These measurements show that vibrational energy redistribution from the antisymmetric to the symmetric $\mathrm{C}-\mathrm{H}$ stretch vibration is very efficient. We have presented evidence that the scattering proceeds via a direct mechanism, making this the first observation of vibrational energy redistribution in a direct molecule-surface collision. Moreover, our measurements demonstrate that bolometric detection in combination with present cw IR light sources is sensitive enough for performing state-to-state scattering experiments, opening new possibilities for studying the dynamics of polyatomic molecules at surfaces.

We thank Alan Luntz for his initial suggestion and the donation of a bolometer and Vincent Boudon for providing spectroscopic data on hot-band transitions of methane. We further thank Fleming Crim for many discussions and gratefully acknowledge financial support provided by the Swiss National Science Foundation (Grant No. 159689) and the Max Planck-EPFL center for molecular nanoscience and technology.

*rainer.beck@epfl.ch

[1] G. O. Sitz, Rep. Prog. Phys. 65, 1165 (2002).

[2] A. W. Kleyn, Chem. Soc. Rev. 32, 87 (2003).

[3] A. Wodtke, D. Matsiev, and D. Auerbach, Prog. Surf. Sci. 83, 167 (2008).

[4] L. Juurlink, D. Killelea, and A. Utz, Prog. Surf. Sci. 84, 69 (2009).

[5] R. Díez Muiño and H.F. Busnengo, Dynamics of GasSurface Interactions: Atomic-Level Understanding of Scattering Processes at Surfaces (Springer, New York, 2013).

[6] H. Chadwick and R. D. Beck, Chem. Soc. Rev. 45, 3576 (2016).

[7] G.-J. Kroes and C. Díaz, Chem. Soc. Rev. 45, 3658 (2016).

[8] K. Golibrzuch, N. Bartels, D. J. Auerbach, and A. M. Wodtke, Annu. Rev. Phys. Chem. 66, 399 (2015).

[9] B. Jiang, M. Yang, D. Xie, and H. Guo, Chem. Soc. Rev. 45, 3621 (2016).
[10] A. M. Wodtke, Chem. Soc. Rev. 45, 3641 (2016).

[11] H. Chadwick and R. D. Beck, Annu. Rev. Phys. Chem. 68, 39 (2017).

[12] B. D. Kay, T. D. Raymond, and M. E. Coltrin, Phys. Rev. B 36, 6695 (1987).

[13] B. D. Kay, T. D. Raymond, and M. E. Coltrin, Phys. Rev. Lett. 59, 2792 (1987).

[14] K. Golibrzuch, J. H. Baraban, P. R. Shirhatti, J. Werdecker, C. Bartels, and A. M. Wodtke, Z. Phys. Chem. 229, 1929 (2015).

[15] G. B. Park, B. C. Krüger, S. Meyer, A. M. Wodtke, and T. Schäfer, Phys. Chem. Chem. Phys. 18, 22355 (2016).

[16] J. Misewich, P. L. Houston, and R. P. Merrill, J. Chem. Phys. 82, 1577 (1985).

[17] T. W. Francisco, N. Camillone III, and R. E. Miller, Phys. Rev. Lett. 77, 1402 (1996).

[18] A. C. Wight, M. Penno, and R. E. Miller, J. Chem. Phys. 111, 8622 (1999).

[19] A. C. Wight and R. E. Miller, J. Chem. Phys. 109, 8626 (1998).

[20] A. C. Wight and R. E. Miller, J. Chem. Phys. 109, 1976(1998).

[21] F. F. Crim, Proc. Natl. Acad. Sci. U.S.A. 105, 12654 (2008).

[22] D. R. Killelea and A. L. Utz, Phys. Chem. Chem. Phys. 15, 20545 (2013).

[23] T. E. Gough, R. E. Miller, and G. Scoles, Appl. Phys. Lett. 30, 338 (1977).

[24] M. Vainio and L. Halonen, Phys. Chem. Chem. Phys. 18, 4266 (2016).

[25] N. V. Vitanov, T. Halfmann, B. W. Shore, and K. Bergmann, Annu. Rev. Phys. Chem. 52, 763 (2001).

[26] H. Chadwick, P. M. Hundt, M. E. van Reijzen, B. L. Yoder, and R. D. Beck, J. Chem. Phys. 140, 034321 (2014).

[27] S. Nave, A. K. Tiwari, and B. Jackson, J. Phys. Chem. A 118, 9615 (2014).

[28] L. B. F. Juurlink, P. R. McCabe, R. R. Smith, C. L. DiCologero, and A. L. Utz, Phys. Rev. Lett. 83, 868 (1999).

[29] P. Maroni, D. C. Papageorgopoulos, M. Sacchi, T. T. Dang, R. D. Beck, and T. R. Rizzo, Phys. Rev. Lett. 94, 246104 (2005).

[30] M. P. Schmid, P. Maroni, R. D. Beck, and T. R. Rizzo, Rev. Sci. Instrum. 74, 4110 (2003).

[31] See Supplemental Material at http://link.aps.org/ supplemental/10.1103/PhysRevLett.120.053402, which includes Ref. [32], for details of the molecular beam conditions, surface sample, fluence curve fitting procedure, and spectroscopic data.

[32] I. Gordon et al., J. Quant. Spectrosc. Radiat. Transfer 203, 3 (2017).

[33] B. L. Yoder, R. Bisson, P. Morten Hundt, and R. D. Beck, J. Chem. Phys. 135, 224703 (2011).

[34] E. B. Wilson, Jr., J. Chem. Phys. 3, 276 (1935).

[35] J. R. Manson, Comput. Phys. Commun. 80, 145 (1994).

[36] A. al Taleb, G. Anemone, D. Farías, and R. Miranda, Carbon 99, 416 (2016).

[37] A. Al Taleb and D. Farías, Phys. Chem. Chem. Phys. 19, 21267 (2017).

[38] D. J. Nesbitt and R. W. Field, J. Phys. Chem. 100, 12735 (1996).

[39] P. Hess and C. B. Moore, J. Chem. Phys. 65, 2339 (1976).

[40] P. Hess, A. H. Kung, and C. B. Moore, J. Chem. Phys. 72, 5525 (1980).

[41] L. Halonen, S. L. Bernasek, and D. J. Nesbitt, J. Chem. Phys. 115, 5611 (2001).

[42] K. G. Prasanna, R. A. Olsen, A. Valdés, and G.-J. Kroes, Phys. Chem. Chem. Phys. 12, 7654 (2010). 